Acta Crystallographica Section D

Biological Crystallography

ISSN 0907-4449

Vicki A. Bamford, ${ }^{a}$ Ola O. Kolade, ${ }^{a}$ Anne E. Osbourn ${ }^{\text {b }}$ and Andrew M. Hemmings $\mathrm{s}^{\mathrm{a}, \mathrm{c} *}$

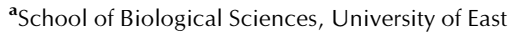
Anglia, Norwich NR4 7TJ, England, 'bainsbury Laboratory, John Innes Centre,

Norwich NR4 7UH, England, and ${ }^{\mathbf{c}}$ School of Chemical Sciences and Pharmacy, University of East Anglia, Norwich NR4 7TJ, England

Correspondence e-mail:

a.hemmings@uea.ac.uk
C) 2004 International Union of Crystallography Printed in Denmark - all rights reserved

\title{
Purification, crystallization and preliminary X-ray diffraction analysis of a fungal saponin-detoxifying enzyme
}

Tomatinase, an extracellular enzyme belonging to family 3 of the glycosyl hydrolases, is produced by the fungal tomato-leaf pathogen Septoria lycopersici and detoxifies the saponin $\alpha$-tomatine. An efficient strategy for purification of the enzyme from fungal culture medium has been developed. Single crystals have been grown by vapour diffusion at $289 \mathrm{~K}$ from $17.5 \%(w / v)$ PEG $4 \mathrm{~K}, 5 \%(v / v)$ 2-propanol and $0.1 \mathrm{M}$ sodium acetate $\mathrm{pH} 4.5$ as precipitant. When cryoprotected at $100 \mathrm{~K}$, these crystals diffract to at least $3.0 \AA$ and belong to space group $P 2_{1} 2_{1} 2$. Based on an estimated molecular weight of $110 \mathrm{kDa}$ for the glycosylated protein and assuming two molecules in the asymmetric unit, the crystals contain approximately $46 \%$ solvent.

\section{Introduction}

Saponins are an important group of glycosylated secondary metabolites that are widely distributed in the plant kingdom. The triterpenoid saponins are found primarily in dicotyledonous plants such as those of the Leguminosae family, but also in some monocots. Steroidal saponins are less common and occur mainly in monocots such as the Liliaceae family. Steroidal glycoalkaloids are found in over 350 plant species, primarily in members of the family Solanaceae, which includes potato and tomato (Hostettmann \& Marston, 1995). Many saponins have antifungal activity and, because they are often present in healthy plants at relatively high levels, may contribute to the resistance of plants to attack by fungi (Morrissey \& Osbourn, 1999). Saponins may therefore be regarded as preformed antimicrobial agents.

There is good evidence that the major mechanism of toxicity of saponins to fungi is associated with their membraneolytic action (reviewed in Morrissey \& Osbourn, 1999). This arises from their ability to form complexes with membrane sterols, resulting in pore formation, electrolyte leakage and loss of membrane integrity. Some fungi have intrinsic resistance to saponins owing to their membrane composition. Other fungi produce specific saponindetoxifying enzymes. The production of extracellular enzymes that detoxify saponins has been documented for a number of plantpathogenic fungi (Morrissey \& Osbourn, 1999). The widespread distribution of saponins in the plant kingdom suggests that the ability to detoxify them may be a common attribute of plant-pathogenic fungi. The saponin-detoxifying enzyme avenacinase, which is produced by the root-infecting pathogen Gaeumannomyces graminis, detoxifies oat root triterpene saponins and has been shown to be a determinant of host range (Bowyer et al., 1995).

A number of fungi that infect tomatoes produce enzymes known as tomatinases that detoxify the steroidal glycoalkaloid $\alpha$-tomatine (reviewed in Morrissey \& Osbourn, 1999). $\alpha$-Tomatine is found in very high concentrations (up to $1 \mathrm{mM}$ ) in the leaves, stems, roots and green fruits of tomatoes, suggesting that it may be important in resistance to potential pathogens. Septoria lycopersici, the causative agent of tomato leaf-spot disease, possesses a constitutive tomatinase which detoxifies $\alpha$-tomatine by removing a single terminal D-glucose moiety (Pegg \& Woodward, 1986; Osbourn et al., 1995; Sandrock et al., 1995). Mutants of $S$. lycopersici that are unable to produce tomatinase trigger elevated defence responses in solanaceous plants (MartinHernandez et al., 2000; Bourab et al., 2002). Interestingly, these experiments and other data suggest that the degradation products of $\alpha$-tomatine hydrolysis are able to interfere directly or indirectly with induced plant defence responses (Martin-Hernandez et al., 2000; Bourab et al., 2002). The high substrate specificity and extracellular nature of saponinases such as tomatinase make them attractive targets for the design of inhibitors to be used in the control of these fungal diseases of plants.

The predicted amino-acid sequence of tomatinase indicates that it belongs to family 3 of the glycosyl hydrolases (Osbourn et al., 1995; Sandrock et al., 1995). This family, which has over 100 members, includes the fungal and bacterial cellobiose-degrading enzymes (Henrissat \& Bairoch, 1996). The sole member of the family for which a crystal structure is 
available is the barley $\beta$-D-glucan exohydrolase (Varghese et al., 1999). This structure has been used to generate comparative (homology) models for other family members representative of the main phylogenetic clusters (Harvey et al., 2000). Members of the cluster including tomatinase are proposed to contain the $(\alpha / \beta)_{8^{-}}$ barrel and $(\alpha / \beta)_{6}$-sandwich structures of domains 1 and 2, respectively, of the barley exohydrolase (and in the same order in the amino-acid sequence). They are, however, significantly larger than the barley enzyme (198 more residues in the case of tomatinase) and as such have additional predicted domains. In addition, the aligned region of tomatinase has only $22 \%$ sequence identity with the barley enzyme and for such highly divergent proteins it is unlikely that homology models can provide an accurate description of the tomatinase structure.

In this paper, we report the development of an efficient purification strategy for tomatinase from Septoria lycopersici culture filtrate and the successful crystallization of the protein. X-ray data have been collected from native protein crystals. Determination of the crystal structure of the enzyme will hopefully provide a structural basis for saponinase-inhibitor design.

\section{Materials and methods}

\subsection{Culture of $S$. Iycopersici}

Petri dishes of potato dextrose agar containing a final concentration of streptomycin of $34 \mu M$ were inoculated with a $1 \mathrm{~mm}^{2}$ section of mycelium from an actively growing $S$. lycopersici colony (Isolate 353-49) provided by the Centraalbureau voor Schimmelcultures, Baarn, The Netherlands. The plates were incubated for 14-20 d in the dark at $295 \mathrm{~K}$. Colonies were homogenized and added to $500 \mathrm{ml}$ Czapek Dox liquid media containing $10 \mathrm{~g} \mathrm{l}^{-1}$ casamino acids and streptomycin to a final concentration of $34 \mu M$. The flasks were incubated for $5 \mathrm{~d}$ at $295 \mathrm{~K}$ with shaking at $300 \mathrm{rev}^{\mathrm{min}}{ }^{-1}$.

\subsection{Protein purification}

Six flasks of $S$. lycopersici liquid culture were filtered and pooled. The supernatant was adjusted to pH 7.0 with $4 M \mathrm{NaOH}$. Protease inhibitors were added to the concentrations indicated: EDTA $(2 \mathrm{~m} M)$, PMSF $(0.05 \mathrm{mM})$, benzamidine hydrochloride $(1 \mu M)$, pepstatin A $(1.5 \mu M)$, phenanthroline $(0.5 \mu M)$, aprotinin $(0.5 \mu M)$ and leupeptin $(2 \mu M)$ (Osbourn et al., 1995). Concentration of the protein was achieved by the addition of $100 \mathrm{~g}$ DE52 anionexchange medium pre-equilibrated with $20 \mathrm{~m} M$ bis-tris propane $\mathrm{pH} 7.0$ per 31 of culture. This was stirred slowly overnight at $277 \mathrm{~K}$ and then allowed to settle. Excess supernatant was removed and the remaining DE52 packed into a XK 26/40 column at $277 \mathrm{~K}$. Fractions were eluted with $20 \mathrm{mM}$ bis-tris propane $\mathrm{pH} 7.0$ plus $1 M \mathrm{NaCl}$. Fractions were assessed for tomatinase activity using a glucose oxidase assay (Sigma) for glucose release from the natural tomatinase substrate, $\alpha$-tomatine, as described by Osbourn et al. (1995) (note that tomatinase-containing samples of culture medium were dialysed using a $10 \mathrm{kDa}$ NMWC membrane to remove free glucose prior to assay) and by SDS-PAGE before pooling and dialysis against water at $277 \mathrm{~K}$.

The concentrated protein was buffered to $20 \mathrm{~m} M$ diethanolamine $\mathrm{pH} 9.0$ by the addition of an appropriate volume of $1 \mathrm{M}$ diethanolamine $\mathrm{pH}$ 9.0. Anion-exchange chromatography was performed on a POROS 20HQ $(4.6 \times 100 \mathrm{~mm})$ column. Fractions were eluted with a linear gradient of 0-400 $\mathrm{m} M \mathrm{NaCl}$. Fractions active against $\alpha$-tomatine were pooled and dialysed against water at $277 \mathrm{~K}$ and then lyophilized. Gel filtration was then carried out using a HiPrep Sephacryl S-200 HR column preequilibrated with $20 \mathrm{~m} M$ sodium phosphate buffer $\mathrm{pH} 6.2$ containing $200 \mathrm{mM} \mathrm{NaCl}$ and eluted with the same buffer.

\subsection{Crystallization and X-ray diffraction analysis}

Tomatinase was concentrated to approximately $10 \mathrm{mg} \mathrm{ml}^{-1}$ using an Amicon diafiltration device at $277 \mathrm{~K}$. The protein sample was filtered through a $300 \mathrm{kDa}$ NMWL filter prior to use in order to remove any aggregates or dust particles that may be present and that could interfere with crystallization. Initial crystallization screening experiments were carried out at both 277 and $281 \mathrm{~K}$ using the screens of Jancarik \& Kim (1991) and Cudney et al. (1994) and the hanging-drop vapour-diffusion technique. Each experiment utilized a $4 \mu \mathrm{l}$ drop containing equal volumes of concentrated protein solution and screen solution. Each hanging drop was equilibrated against a $700 \mu \mathrm{l}$ reservoir of screen solution. Crystals were observed after several days from an optimized condition containing $17.5 \%(w / v)$ PEG 4K, 5\%(v/v) 2-propanol and $0.1 M$ sodium acetate $\mathrm{pH} 4.5$ at $277 \mathrm{~K}$. Crystals were cryoprotected by transfer to a solution containing 20\%( $w / v)$ PEG 4K, $0.1 M$ sodium acetate $\mathrm{pH} 4.5$ and $15 \%(v / v)$ ethylene glycol.
X-ray diffraction data were collected on Station 9.6 of the SRS (Daresbury Laboratory) using an ADSC Quantum $4 \mathrm{CCD}$ detector and a wavelength of $0.87 \AA$. The data were collected at $100 \mathrm{~K}$ using an oscillation angle of $1^{\circ}$ per image. Data processing was performed with the $H K L$ program suite by means of the programs $D E N Z O$ and SCALEPACK (Otwinowski \& Minor, 1997). Structure-factor amplitudes were derived from the observed reflection intensities and self-rotation functions were calculated using programs from the $C C P 4$ program suite (Collaborative Computational Project, Number 4, 1994). The original structure-factor data were insufficient to unambiguously determine the space group of the tomatinase crystals and so axial reflections were collected to medium resolution from a large single crystal cryocooled to $100 \mathrm{~K}$ and mounted on a Rigaku RU-200HB copper rotating-anode X-ray generator equipped with Osmic mirrors and an R-AXIS IV detector.

\section{Results and discussion}

A simple three-step purification protocol was developed to maximize the yield of tomatinase from fungal cell-culture medium (Fig. 1). The yields and specific activities of tomatinase from this procedure are presented in Table 1. A major improvement over previously published methods (e.g. Osbourn et al., 1995; Sandrock et al., 1995) involved the use of a batch anion-exchange protein-concentration step in place of the use of ammonium sulfate as precipitant. The final yield was $1.0 \mathrm{mg}$ of protein per litre of fungal culture medium, with a purification factor of 15.5. The increase in total activity of the tomatinase preparations following the concentration step of the purification procedure is interesting and may have resulted from the removal of a high-

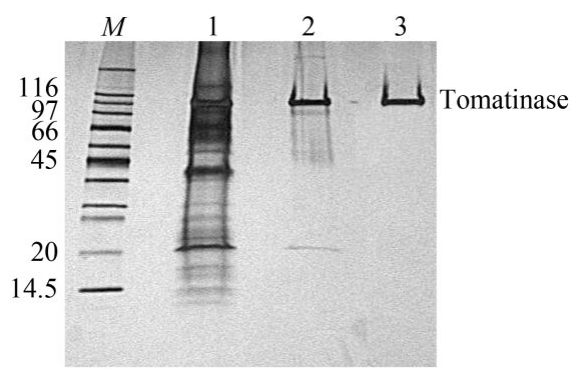

Figure 1

A silver-stained 10-20\% gradient SDS-PAGE gel showing the progress of purification of tomatinase. Lane $M$, molecular-weight markers (kDa); lane 1 , culture filtrate (100 ng total protein); lane 2, pooled fractions after POROS HQ anion-exchange chromatography (10 ng total protein); lane 3, purified tomatinase (10 $\mathrm{ng}$ protein) 
Table 1

Purification statistics for tomatinase.

Figures quoted are per litre of culture medium.

\begin{tabular}{|c|c|c|c|}
\hline Purification stage & $\begin{array}{l}\text { Total protein } \\
(\mathrm{mg})\end{array}$ & 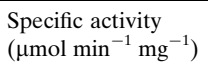 & $\begin{array}{l}\text { Total activity } \\
\left(\mu \mathrm{mol} \min ^{-1}\right)\end{array}$ \\
\hline Culture filtrate & 43 & 10 & 430 \\
\hline Concentration & 20 & 40 & 800 \\
\hline Anion exchange & 2.0 & 150 & 300 \\
\hline Gel filtration & 1.0 & 155 & 155 \\
\hline
\end{tabular}

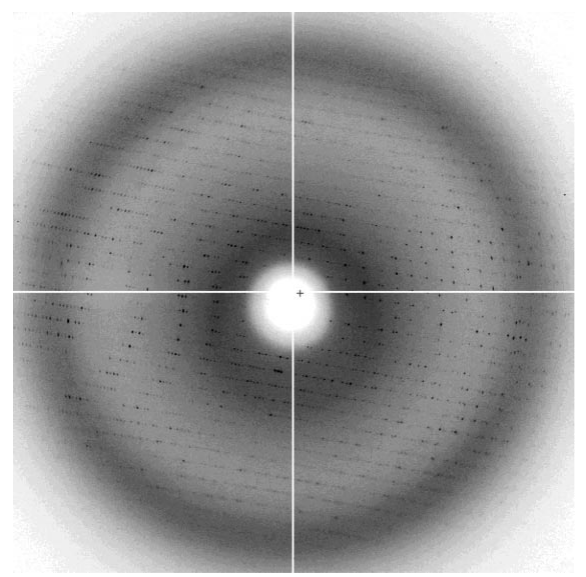

Figure 2

$\mathrm{X}$-ray diffraction pattern from a crystal of tomatinase.

molecular-weight factor which interferes with either tomatinase or glucose oxidase activity or both. Further investigation will be necessary to identify this component.

Despite the extensive glycosylation of the protein, crystallization trials using the purified enzyme yielded crystals from a crystallization solution containing a mixture of $17.5 \%(w / v)$ PEG $4 \mathrm{~K}$ and $5 \%(v / v)$ 2-propanol as precipitant. Optimization of the crystallization conditions gave single crystals of typical dimensions $100 \times 50 \times 20 \mu \mathrm{m}$. When protein from dissolved crystals was analyzed by SDS-PAGE, a single band at an apparent molecular weight of $110 \mathrm{kDa}$ was detected on staining. These samples were also active against $\alpha$-tomatine substrate.

The crystals were cryoprotected in $20 \%(w / v)$ PEG 4K, $0.1 M$ sodium acetate $\mathrm{pH} 4.5$ and $15 \%(v / v)$ ethylene glycol prior to flash-freezing in an $\mathrm{N}_{2}$-gas stream. Diffraction data collection from single crystals
Table 2

Native X-ray data-collection statistics for tomatinase.

Values in parentheses refer to data in the highes resolution shell (3.11-3.0 ^).

\begin{tabular}{ll}
\hline Beamline & SRS Station 9.6 \\
Wavelength $(\AA)$ & 0.87 \\
Unit-cell parameters $\left(\AA{ }^{\circ}\right)$ & $a=57.4, b=149.3$, \\
& $c=240.3, \alpha=90$, \\
& $\beta=90, \gamma=90$ \\
Resolution range $(\AA)$ & $20-3.0$ \\
$R_{\text {sym }} \dagger(\%)$ & $14.7(31.7)$ \\
$\langle I\rangle /\langle\sigma(I)\rangle<3(\%)$ & $29.1(53.5)$ \\
Completeness $(\%)$ & $93.3(85.9)$ \\
Total observations & 314896 \\
Independent reflections & 42819 \\
\hline
\end{tabular}

$\dagger R_{\mathrm{sym}}=\sum \sum\left|I_{i}-\langle I\rangle\right| / \sum I_{i}$, where $\langle I\rangle$ is the average of symmetry-equivalent reflections and the summation extends over all observations for all unique reflections.

(Fig. 2) using synchrotron radiation gave a native data set to a maximum resolution of $3.0 \AA$ (Table 2). Autoindexing and consideration of systematically absent reflections reveal that the crystals belong to space group $P 2{ }_{1} 2{ }_{1} 2$ or $P 222_{1}$, with unit-cell parameters $a=57.4, b=149.3, c=240.3 \AA$, $\alpha=\beta=\gamma=90^{\circ}$. Additional axial reflections collected to medium resolution on an inhouse X-ray detector system revealed the true space group to be $P 2_{1} 2_{1} 2$. Assuming that there are two molecules per asymmetric unit and a protein molecular weight of $85 \mathrm{kDa}$ (as calculated from the amino-acid sequence), $V_{\mathrm{M}}$ is $2.99 \AA^{3} \mathrm{Da}^{-1}$, corresponding to a solvent content of $58.5 \%$. If a molecular weight of $110 \mathrm{kDa}$ (glycoprotein molecular weight as determined by SDSPAGE) is used, $V_{\mathrm{M}}$ falls to $2.31 \AA^{3} \mathrm{Da}^{-1}$ and the solvent content falls to $46.3 \%$. However, calculation of self-rotation functions using the $C C P 4$ program suite failed to identify a clear non-crystallographic twofold axis. Molecular replacement using a variety of search models based on the barley $\beta$-Dglucan exohydrolase was also unsuccessful. Crystallographic cells deduced from diffraction data collected from a number of crystals of the protein were isomorphous, suggesting that the MIR method may be appropriate for phase determination. The search for suitable heavy-atom derivatives is in progress.

We gratefully acknowledge financial support from the BBSRC under grant 83/ B09174. We are also acknowledge access to Station 9.6 at the SRS, Daresbury Laboratory.

\section{References}

Bourab, K., Melton, R., Peart, J., Baulcombe, D. \& Osbourn, A. (2002). Nature (London), 418, 889-892.

Bowyer, P., Clarke, B. R., Lunness, P., Daniels, M. J. \& Osbourn, A. E. (1995). Science, 267, 371-374.

Collaborative Computational Project, Number 4 (1994). Acta Cryst. D50, 760-763.

Cudney, R., Patel, S., Weisgraber, K., Newhouse, Y. \& McPherson, A. (1994). Acta Cryst. D50, 414-423.

Harvey, A. J., Hrmova, M., De Gori, R., Varghese, J. N. \& Fincher, G. B. (2000). Proteins, 41, 257269.

Henrissat, B. \& Bairoch, A. (1996). Biochem J. 316, 695-696.

Hostettmann, K. A. \& Marston, A. (1995) Chemistry and Pharmacology of Natural Products. Cambridge University Press.

Jancarik, K. \& Kim, S.-H. (1991). J. Appl. Cryst. 24, 409-411.

Martin-Hernandez, A. M., Dufresne, M., Hugouvieux, V., Melton, R. \& Osbourn, A. E. (2000). Mol. Plant Microbe Interact. 13, 1301-1311.

Morrissey, J. P. \& Osbourn, A. E. (1999). Microbiol. Mol. Biol. Rev. 63, 708-724.

Osbourn, A. E., Bowyer, P., Lunness, P., Clarke, B. \& Daniels, M. (1995). Mol. Plant Microbe Interact. 8, 971-978.

Otwinowski, Z. \& Minor, W. (1997). Methods Enzymol. 276, 307-326.

Pegg, G. F. \& Woodward, S. (1986). Physiol. Mol. Plant Pathol. 28, 187-201.

Sandrock, R. W., Della Penna, D. \& Van Etten, H. D. (1995). Mol. Plant Microbe Interact. 8, 960-970.

Varghese, J. N., Hrmova, M. \& Fincher, G. B. (1999). Structure, 7, 179-190. 